\title{
Trasplante facial generalidades
}

\author{
Jefferson Gerardo Méndez-Cárdenas* \\ Daniela Nieto-Fernández * \\ María Camila Erazo-Arteaga* \\ Daniel Mauricio Pineda-López* \\ Diana Yeraldín Quintero-Cuervo* \\ Paola Andrea Santamaría-Losada* \\ Carlos Eduardo Ruiz-Moreno** \\ Miguel Angel Nieto-Bayona** \\ Sergio Alejandro Leuro-Torres** \\ Miguel Ángel Gómez-Rueda***
}

\begin{abstract}
*Médico y Cirujano. Integrante Semillero de Investigación de Cirugía Plástica de la Universidad Nacional de Colombia. Bogotá D.C. Cundinamarca. Colombia.

**Estudiante de Medicina XII semestre. Universidad Nacional de Colombia. Integrante Semillero de Investigación de Cirugía Plástica de la Universidad Nacional de Colombia. Bogotá D.C. Cundinamarca. Colombia.

***Estudiante de Medicina X semestre. Universidad Nacional de Colombia. Integrante Semillero de Investigación de Cirugía Plástica de la Universidad Nacional de Colombia. Bogotá D.C. Cundinamarca. Colombia.

Correspondencia: Dr. Jefferson Gerardo Méndez Cárdenas. Dirección: Cra 10 \# 81 B 92 sur este. Bogotá. Cundinamarca. Colombia. Teléfono: 3164317820. Dirección electrónica:jgmendezc@unal.edu.co
\end{abstract}

\section{Resumen}

El trasplante facial es un alotrasplante compuesto vascularizado que busca la reconstrucción de defectos masivos faciales permitiendo la reinserción social del receptor, generando un impacto positivo sobre su calidad de vida. El objetivo de este artículo fue realizar una revisión de la literatura sobre generalidades del trasplante facial, enfocándose en los cuidados pre, intra y postoperatorios concernientes a la temática. La búsqueda bibliográfica se realizó desde el 13 de septiembre del 2018 hasta el 31 de marzo del 2019 utilizando los términos MESH “Face Transplant” y “Face Transplantation”. Aplicando los criterios de inclusión y exclusión se obtuvieron 43 artículos sobre los cuales se desarrolló la revisión. El trasplante facial es un procedimiento nuevo en el cual hay muchas áreas por explorar, sin embargo, es una alternativa prometedora a los métodos utilizados para la reconstrucción de lesiones faciales complejas, que se optimizará a medida que se extienda su uso. MÉD.UIS.2020;33(3): 29-36.

Palabras clave: Trasplante facial. Trasplante alogénico. Periodo preoperatorio. Periodo postoperatorio. Bioética.

\section{Facial trasplant generalities}

\section{Abstract}

The facial transplant is a vascularized compound allogeneic transplant that seeks the reconstruction of massive facial defects allowing the social reintegration of the recipient, generating a positive impact on their quality of life. The objective of this article was to review the literature on generalities of facial transplantation, focusing on the pre, intra and postoperative care regarding the subject. The bibliographic search was performed from September 13, 2018 to March 31, 2019 using the MESH terms "Face Transplant" and "Face Transplantation". Applying the inclusion and exclusion criteria, 43 articles were obtained on which the review was developed. Facial transplantation is a new procedure in which there are many areas to explore, however, it is a promising alternative to the methods used for the reconstruction of complex facial injuries, which will be optimized as its use expands. MÉD.UIS.2020;33(3): 29-36.

Keywords: Facial transplant. Allogenic transplant. Preoperative period. Postoperative period. Bioethics. 
¿Cómo citar este artículo?: Méndez-Cárdenas JG, Nieto-Fernández D, Erazo-Arteaga MC, PinedaLópez DM, Quintero-Cuervo DY, Santamaría-Losada PA, Ruiz-Moreno CE, Nieto-Bayona MA, Leuro-Torres SA, Gómez-Rueda MA. Trasplante facial generalidades. MÉD.UIS.2020;33(3):29-36. doi: 10.18273/revmed.v33n3-2020003

\section{Introducción}

El trasplante facial (TF) es un procedimiento quirúrgico en el cual, por medio de técnicas de microcirugía, se realiza la reconstrucción de defectos masivos faciales para restaurar la funcionalidad y morfología del rostro. Se realizó por primera vez en Francia en el año 2005, por los doctores Bernard Devauchelle y Jean-Michel Dubernary y desde entonces ha abierto las puertas a una nueva era en el área reconstructiva'.

Hasta el año 2016 se encontraron 37 casos reportados realizados en Europa, Asia y Estados Unidos (no se tiene registro de trasplantes faciales realizados en Latinoamérica). De estos casos, 15 corresponden a trauma balístico,10 a quemaduras, 4 a neurofibromatosis, 3 a ataques de animales, 2 a lesiones vasculares, 2 a trauma facial y 1 a secuelas de cáncer y radioterapia'. Adicionalmente, en la literatura se ha reportado que concomitante a su patología de base, los pacientes receptores presentan trastornos psiquiátricos, entre los cuales predominan la ansiedad y la depresión, en su mayoría secundarias al aislamiento social y la discriminación ${ }^{2,3}$.

Actualmente, el método de elección utilizado para la reconstrucción de lesiones faciales complejas es el colgajo libre, que, si bien es una buena alternativa, ofrece resultados subóptimos dada la complejidad y diversidad de tejidos a nivel facial ${ }^{4-10}$. Teniendo en cuenta lo anterior, el trasplante facial se convierte en una buena opción para la intervención de este tipo de lesiones buscando mejores desenlaces. Cabe resaltar que, aunque no es un procedimiento vital, estudios han reportado una mejora significativa en la calidad de vida de los pacientes ${ }^{11,12}$.

Uno de los puntos más importantes del procedimiento es la evaluación preoperatoria del paciente, la cual debe ser llevada a cabo por un equipo multidisciplinario y en donde se hará evaluación de los riesgos y beneficios de la intervención, teniendo en cuenta las implicaciones de recibir un trasplante facial (entre las cuales se destaca el inmunocompromiso), así como las posibles complicaciones posteriores a este, individualizadas para cada paciente ${ }^{7,10,11}$.

Durante la búsqueda en las bases de datos no se encontró en la bibliografía artículos científicos ni libros que compilaran el TF en la literatura colombiana, por ende el objetivo de este artículo es realizar una revisión de la literatura sobre generalidades del trasplante facial, enfocándose en los cuidados pre, intra y postoperatorios concernientes a la temática.

\section{Metodología de búsqueda}

Se realizó una búsqueda exhaustiva de la información disponible en los artículos publicados entre marzo del 2008 y marzo del 2018. Esta se realizó desde el 13 de septiembre del 2018 hasta el 31 de marzo del 2019 utilizando los términos MESH "Face Transplant" y "Face Transplantation" en las bases de datos "Pubmed, Scielo, Lilacs, Inbiomed, Clinical key, Ovid, Embase, ScienceDirect, BioMedCentral, Google Scholar y Medline", obteniendo un total de 120924 documentos de los cuales se seleccionaron aquellos artículos que tenían los términos MESH en el título o en el "abstract" filtrando así 55065 documentos.

De estos últimos se seleccionaron aquellos que dataran del año 2008 hasta el 31 de marzo del 2018, que incluyeran aspectos bioéticos y procedimientos durante el periodo preoperatorio del trasplante, así como aquellos artículos que detallaran técnicas y planeamientos intraoperatorios, esquemas farmacológicos de inmunosupresión, métodos de rehabilitación, calidad de vida postoperatoria y costos del procedimiento quirúrgico. Tras esto, se descartaron 55065 documentos y resultaron 402 artículos.

Finalmente, se excluyeron 359 publicaciones como cartas al editor, comunicados, o documentos que trataran temas sobre procedimientos reconstructivos diferentes al trasplante facial, como aspectos sociológicos e inmunes sobre este procedimiento. Por lo que 43 artículos resultantes fueron seleccionados para esta revisión. 


\section{Desarrollo del tema}

\section{Periodo preoperatorio}

En principio previo a un procedimiento como lo es el $\mathrm{TF}$, es importante realizar un análisis preoperatorio: multidisciplinario y exhaustivo el cual tenga en cuenta la estructura ósea, el estado de los tejidos blandos faciales, las necesidades funcionales del paciente y los aspectos ortognáticos; esto se lleva a cabo principalmente con estudios de imagen como modelos 3D, resonancia magnética, angiografía, tomografía y ultrasonografía en modalidad Doppler ${ }^{13,14}$. Debe hacerse un análisis cefalométrico y evaluar los predictores de vía aérea difícil teniendo en cuenta los cambios en la anatomía de los pacientes, y a las múltiples intervenciones reconstructivas previas; al igual que reservar una cantidad suficiente de unidades de glóbulos rojos ${ }^{15}$.

\subsection{Criterios de selección de donante y receptor}

\subsubsection{Donante}

Se toma en consideración pacientes con muerte cerebral, sin patologías cardíacas y con características similares al receptor como: edad, sexo, talla, peso, color de piel, color de cabello, dimensiones craneofaciales y grupo sanguíneo $A B O$ idéntico ${ }^{1,3,13}$. Es importante el desarrollo de un algoritmo logístico para la adquisición de tejidos de un donante como el propuesto por "The New York University"; donde se prioriza la seguridad del equipo quirúrgico, la integridad del órgano sólido y el tiempo de isquemia reducido del aloinjerto facial ${ }^{16}$.

\subsubsection{Receptor}

En las bases de datos consultadas no se ha realizado hasta la fecha actual un consenso mundial respecto a los criterios de la selección del receptor de selección del receptor, sin embargo, la "American Society of Plastic Surgeons" y la "American Society of Microsurgery", recomiendan el trasplante solo en pacientes con desfiguración severa $(\geq 25 \%$ del total de la cara incluyendo subunidades centrofaciales) cuando la reconstrucción convencional no ha sido satisfactoria. Entre los receptores se han descrito pacientes con trauma balístico, quemaduras, neurofibromatosis, ataques por animales y secuelas por cáncer ${ }^{17,18}$. La inclusión de pacientes con ceguera total bilateral es un tema en discusión y se recomienda cuando la recuperación de la visión es una opción dentro del procedimiento quirúrgico. Se requiere que el paciente cuente con suficiente reserva de tejido autólogo en caso de rechazo y falla en la integración, siendo posible su uso en procedimientos contingentes con miras a salvaguardar la vida del receptor. Algunos autores señalaron como contradictorias las dos premisas previas, puesto que agotar las posibilidades de reconstrucción significa necesariamente hacer uso del tejido autólogo que muchas áreas donantes tienen para ofrecer ${ }^{17,18}$.

Hay instituciones que han elaborado documentos relacionados con la selección de los receptores y su implicación. Por ejemplo, el "Brigham and Women's Hospital" desarrolló un protocolo de trasplante facial donde enfatizó en el método de referencia de los pacientes, la posibilidad de seguimiento y los controles médicos". Por su parte, "The Cleveland Clinic FACES Score" permitió predecir los resultados y el pronóstico del procedimiento, dando importancia a la historia psicosocial y al estado inmunológico previo. Porúltimo, se debe determinar silos beneficios superan los riesgos y el costo para el paciente, así como para el sistema de salud a largo plazo ${ }^{17,19}$. Este procedimiento está contraindicado en pacientes con baja adherencia al programa de rehabilitación y al manejo farmacológico, personas sin soporte social, con una enfermedad maligna activa y en quienes no se han agotado otras posibilidades de reconstrucción $n^{17,20}$.

\subsection{Indicaciones en adultos}

Se considera a pacientes con deformidades complejas severas por desfiguración traumática o posteriores a la resección de tumores, las cuales comprometen la apariencia, funcionalidad, bienestar del paciente y que no sea posible restaurarlas mediante métodos convencionales. La intervención debe cumplir con los principios de la microcirugía, trasplante de órganos, así como terapia de inmunosupresión que evite el rechazo ${ }^{21,22}$. Es importante la presencia de un equipo multidisciplinario que incluya cirujanos reconstructivos, especialistas en trasplantes, odontólogos, psicólogos, y terapeutas de lenguaje, quienes determinen si está indicado el procedimiento $^{23}$.

\subsection{Indicaciones en niños}

Las indicaciones y justificaciones fueron controvertidas, dado que la duración de la terapia inmunosupresora y la morbilidad secundaria no se conocen con certeza. Los posibles donantes son 
Méndez-Cárdenas JG, Nieto-Fernández D, Erazo-Arteaga MC, Pineda-López DM,

Quintero-Cuervo DY, Santamaría-Losada PA, Ruiz-Moreno CE, Nieto-Bayona MA, Leuro-

pacientes con anencefalia que fallecieron al nacer o con diagnóstico confirmado de muerte cerebral y en lo posible, deben ser pocos años mayor al receptor ${ }^{24}$. Bernard y colaboradores manifiestan que en términos fisiológicos los tejidos implicados son similares a los de un adulto, y, por lo tanto, el trasplante en niños es viable. A partir de esto se proponen una serie de posibles indicaciones pediátricas (que son similares a las de adultos) incluyendo pacientes quemados con secuelas faciales muy graves, trauma con defectos de avulsión en área peribucal, hendiduras faciales múltiples, neoplasias, defectos creados por la resección terapéutica de tumores, malformaciones linfáticas o arteriovenosas y neurofibromatosis tipo 1. Cabe aclarar que todas ellas aún se encuentran permeadas por muchos interrogantes (riesgo beneficio, consecuencias de la retracción cicatricial en el crecimiento mandibular) aún sin respuesta, pues sin duda existe un gran vacío en la literatura en este aparte 25,26 .

El procedimiento está contraindicado en portadores de $\mathrm{VIH}$, pacientes con antecedente de neoplasias recientes y trastornos psiquiátricos no resueltos ${ }^{26}$. El receptor debe manifestar el deseo de la intervención y ser capaz de comprender sus complicaciones ${ }^{24}$. Hasta el momento de la búsqueda bibliográfica no se había reportado el primer caso de TF pediátrico en el mundo, sin embargo, es un procedimiento éticamente razonable, ya que respeta la autonomía del paciente considerando mejorar la calidad de sus años de vida más que aumentar su cantidad ${ }^{26}$. La técnica quirúrgica es igual que la descrita en adultos, teniendo en cuenta que los niños presentan una mayor y más rápida regeneración de tejidos. ${ }^{25} \mathrm{El}$ rango de edad en el cual el receptor tiene la capacidad de tomar la decisión de someterse al procedimiento no se había establecido, pero se sabe que la cronicidad de su patología es directamente proporcional a la madurez para reconocer sus limitaciones y las fallas en las intervenciones médicas previas ${ }^{24}$.

En un estudio realizado por Marchac se buscaron pacientes pediátricos que pudieran ser candidatos para TF, logrando encontrar tres posibles pacientes con las siguientes condiciones médicas: quemadura de tercer grado en toda la cara, síndrome SturgeWeber y neurofibromatosis tipo 1; a quienes se les realizaron previamente múltiples procedimientos quirúrgicos. En dichos pacientes se evaluaron las posibles intervenciones con alotrasplante, compuesto vascularizado que podrían realizarse a cada uno, evidenciando la viabilidad que tiene el TF en beneficio del paciente comparado con las técnicas hasta hoy utilizadas ${ }^{24}$. Aun cuando la terapia inmunosupresora puede traer múltiples desventajas en un cuerpo en etapa de crecimiento, los niños son los que mejor recuperación nerviosa y mejores resultados funcionales podrían tener del TF²5,26.

\subsection{Aspectos bioéticos}

El uso del TF es controversial, aún no existe un consenso internacional que asuma una postura clara frente a la incógnita sobre si deben asumir los riesgos del TF dado que no es un procedimiento vital para el paciente. Desde la óptica bioética, en la práctica médica se deben tener en cuenta los 4 principios básicos: autonomía, no maleficencia, beneficencia y justicia $^{27}$. La autonomía se ve reflejada en la información brindada al paciente, que debe ser veraz y completa, enfatizando en los riesgos al realizar el consentimiento informado. En cuanto a los principios de beneficencia y no maleficencia, es necesario un estudio prequirúrgico con un equipo multidisciplinario encargado de tomar todas las medidas para evitar al máximo las complicaciones y permitir que se obtengan los mejores resultados. Por último, la justicia hace alusión a la distribución de recursos (teniendo en cuenta el alto costo que este tipo de procedimiento representa para los sistemas de salud) y selección del candidato al procedimiento $0^{20,23}$.

\subsection{Modelos experimentales}

Es evidente que este procedimiento posee una complejidad demasiado alta, lo cual, combinado con la escasez con la que se lleva a cabo actualmente, hace difícil obtener curvas de aprendizaje seguras, limitando su enseñanza, implementación y difusión. Ante estos limitantes, la cirugía experimental en modelos animales surge como una alternativa no sólo para estudiar la respuesta fisiológica tisular, sino también para aportar experiencia al personal en formación. Se han identificado distintos modelos posibles, incluyendo murinos, conejos, caninos; pero por su similitud anatómica e incluso inmunológica los cerdos se posicionan como la mejor opción, inclusive ya se cuentan experiencias con porcinos para trasplantes de subunidades faciales. Se han realizado ya ensayos dentro de los cuales se hacen TF in vivo en esta especie, obteniendo resultados favorables y escenarios en los que se pueden analizar los ámbitos pre, intra y postoperatorios ${ }^{28,29}$. 


\section{Periodo intraoperatorio}

\subsection{Procedimiento}

Este proceso tiene una duración aproximada de 20 horas y en cuanto al uso de anestésicos inhalatorios, se conoce que el sevoflurano (con concentración alveolar mínima de 0.8 a 1.8) ha mostrado efectos benéficos en la microcirculación al reducir la extravasación de plasma disminuyendo el edema tisular comparado con anestésicos intravenosos. Además, parece proteger las células endoteliales de daños por isquemia-reperfusión ${ }^{15,22}$.

Se verifica la perfusión del aloinjerto usando angiografía con verde de indocianina, y estimulacion nerviosa ${ }^{13}$. Se debe tener en cuenta la anatomía de la arteria facial realizando un mapeo, identificando sus ramas y variaciones y detallando las regiones que serán intervenidas. El aloinjerto facial se diseca de forma cráneo caudal de lateral a medial. Las ramas del nervio facial se van disecando medial a la parótida; para después unirlas a las ramas receptoras. La glándula parótida sólo se incluye cuando hay defectos de volumen ${ }^{30}$. Las etapas del procedimiento se describen a continuación. (Ver imagen 1)

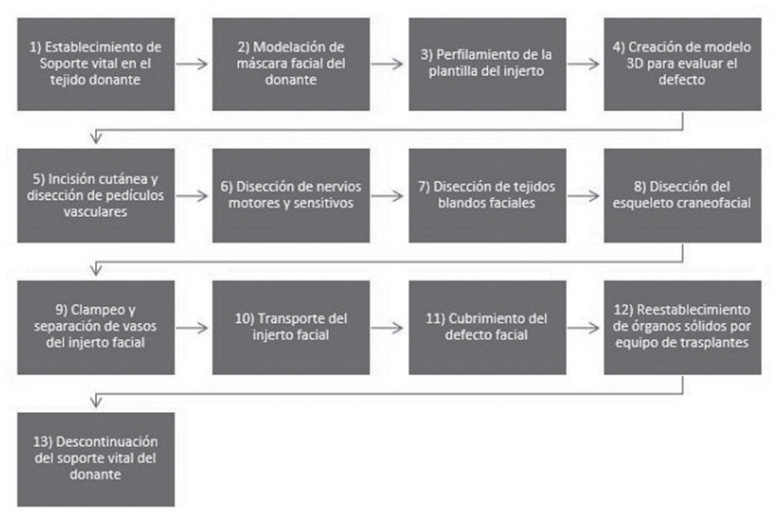

Imagen 1. Secuencia operatoria del TF.

Fuente: Modificado del artículo de Seok Chan Eun ${ }^{30}$.

\section{Periodo postoperatorio}

\section{1 Inmunosupresión}

Es necesario un esquema de inmunosupresión de por vida para asegurar el éxito del TF y evitar el rechazo del huésped a los tejidos injertados, ya sea agudo o crónico. El rechazo agudo es caracterizado por la erupción e hinchazón durante el primer año. La persistencia de estos episodios concomitantes a rigidez de la piel, capilares engrosados y cicatrices se asocian a rechazo crónico del injerto ${ }^{4}$.
Para evitar este rechazo existen dos tipos de terapia de inmunosupresión: está la terapia de inducción cuyo objetivo es evitar el rechazo agudo y generalmente se usa una combinación de timoglobulina, rituximab y prednisona. Los dos primeros interfieren con la respuesta humoral y de forma indirecta con la actividad de linfocitos $\mathrm{T}^{30}$. El otro tipo de terapia es de mantenimiento, y su objetivo es evitar el rechazo crónico y así la falla del aloinjerto, para esta se utilizan terapias combinadas con esteroides (prednisona), inhibidores de la calcineurina (tacrolimus), y de la proliferación de células B y T (micofenolato) ${ }^{1,7}$. Regularmente se obtienen niveles séricos de creatinina y tacrolimus ${ }^{13,17}$ por el riesgo de presentar falla renal secundaria ${ }^{31}$; si llegase a suceder puede suspenderse e iniciar manejo con sirolimus ${ }^{30}$.

Se ha visto un incremento de 2 a 4 veces en la recurrencia de tumores de órgano sólido por la inmunosupresión, aunque se necesitan más estudios que determinen si el riesgo mencionado es extrapolable en pacientes que se sometan al procedimiento con historia previa de alguna patología maligna ${ }^{17,18}$. También aumenta los riesgos de hipertensión arterial, hiperglucemia, alteraciones intestinales e infecciones oportunistas ${ }^{1,4,30}$; éstas últimas son comunes, por ello la importancia de la profilaxis antimicrobiana que se realiza con con trimetoprim-sulfametoxazol, valganciclovir y fluconazol ${ }^{10}$.

Por lo anterior, la terapia de inmunosupresión estricta de por vida es la mayor dificultad a la que se ven enfrentados los pacientes que han recibido TF. Actualmente, la meta es lograr una tolerancia que sea adecuada y duradera frente al trasplante mediante la investigación e innovación en el campo de la terapia inmunosupresora ${ }^{30}$.

\subsection{Rehabilitación}

En el postoperatorio el cerebro no reconoce la nueva cara como suya, lo que sumado al tiempo de reposo potencia la pérdida de habilidades físicas, mentales y sociales ${ }^{22}$. No existen pautas claras para el proceso de rehabilitación, pues cada caso se ha trabajado de manera individual con las consecuentes variaciones en métodos, resultados y tiempo de recuperación. A pesar de dichas diferencias, se ha encontrado una secuencia de recuperación que inicia en la parte sensorial y finaliza en la motora ${ }^{20,22,32-34}$. Aunque se dispone de poca literatura se han sentado bases que parecen ser claves como 
el inicio temprano del proceso ( 2 meses posterior a cirugía), la selección de ejercicios específicos para cada paciente, la estimulación eléctrica funcional y la interdisciplinariedad del equipo de trabajo que incluya cuidado psicológico, espiritual y social ${ }^{12,20,35,36}$. Es indispensable la educación del medico de atención primaria para el reconocimiento de signos de rechazo del trasplante en lugares apartados a los centros de referencia ${ }^{37}$.

\subsection{Calidad de vida}

Hay pocos estudios que evalúen los resultados psicológicos y funcionales de la intervención ${ }^{38}$. Hasta el momento se han realizado tres informes sobre pruebas psicológicas cuantitativas y se ha demostrado que este procedimiento les brinda a los pacientes la oportunidad de retomar sus vidas, recuperar la autoestima, disminuir la depresión, disminuir el abuso verbal que experimentan en público y permitirles la reintegración social; todo ello mejora significativamente su salud mental ${ }^{27,39,40}$. Además, brinda salud física por mejoramiento de la capacidad olfativa, deglutoria, respiratoria, del habla y la sensibilidad facial. Sin embargo, el aspecto que concierne a los retos médicos y dificultades permanece en la sombra, y aún se cuestiona el riesgo y beneficio de este procedimiento considerando la dependencia a las instituciones médicas y a los fármacos a los que estos pacientes quedan sometidos posterior a la cirugía ${ }^{40}$.

\subsection{Complicaciones}

Se han reportado la aparición de enfermedades infecciosas tempranas en el primer mes (por microorganismos oportunistas como Citomegalovirus, virus del Herpes Simple, Pseudomonas Aeruginosa) y enfermedades tardías (principalmente asociadas a Micobacterias) ${ }^{33}$. De igual manera, se ha encontrado disminución de la tasa de filtración glomerular, rechazo agudo desde el primer día hasta los 7 años posteriores al trasplante, y trastornos linfoproliferativos en el $3,8 \%$ de los pacientes trasplantados con una tasa de mortalidad de 11,5\% (para el año 2016 cinco pacientes habían muerto tras realizarse este procedimiento); además de múltiples efectos secundarios de la terapia inmunosupresora requiriendo terapia de mantenimiento con dosis altas de esteroides ${ }^{21,26,38,41}$.

En los casos en los que el aloinjerto incluyó la región auricular, la estenosis y curvatura del conducto auditivo llevó a sensación de ocupación y obstrucción, además de asociarse a la aparición de otitis externa crónica, acumulación de cerumen y disfunción auditiva ${ }^{11}$. En la mayoría de los pacientes usualmente se necesitan reintervenciones para corregir defectos derivados de la piel o de tejidos blandos redundantes, buscando mejorar el desenlace estético y funcional. Al considerar reintervenciones, en especial por motivos estéticos, es importante sopesar el riesgo de comprometer funcionalmente al tejido injertado en comparación con los beneficios en forma y función de las cirugías secundarias ${ }^{21}$.

\section{Costos}

Se encontraron 2 estudios comparando los costos de la reconstrucción facial convencional y el TF. El primero comparó los costos de ambos métodos en un mismo paciente. Se realizó una recolección de datos sobre costos pretrasplante (reconstrucción convencional) desde noviembre 8 de 2004 a diciembre 8 de 2008 (1491 días),y se recolectaron datos peritrasplante desde diciembre 8 de 2008, cuando se realizó la cirugía, hasta febrero 5 de 2009, día del egreso (58 días). Adicionalmente se obtuvieron datos de costos postrasplante desde el día del egreso hasta noviembre 27 de 2009 (295 días). Debe destacarse que los costos peritrasplante incluyen tanto costos del procedimiento quirúrgico como de la hospitalización subsecuente. Los costos directos se calcularon en \$206 646 dólares pretrasplante, \$232 893 peritrasplante y \$74 236 postrasplante, incluyendo costos intrahospitalarios y atención ambulatoria. Existió una diferencia neta de \$26 247 dolares, lo que implica un aumento de $12.7 \%$ al recurrir al TF, frente a las técnicas convencionales. Lo anterior teniendo en cuenta, que no se incluyeron los costos asociados a la inmunosupresión de por vida requerida por el paciente ${ }^{42,43}$.

En segundo lugar, se encontró un estudio comparativo que incluyó 73 pacientes sometidos a reconstrucción convencional, frente a 4 pacientes llevados a TF. En este estudio se evidenció que para ambos grupos el componente de mayor valor fue el de costos intrahospitalarios, el cual incluye el valor de las intervenciones quirúrgicas y estancia hospitalaria. Adicionalmente los costos durante el primer año fueron significativamente mayores en el TF (media, \$337 360 dolares; mediana, \$313 068) que en el grupo de la reconstrucción convencional (media, \$70 230; mediana, \$64 451). Se recalca que, al ser un procedimiento innovador, los costos del 
TF son elevados, pero se espera que disminuyan conforme se extienda la práctica ${ }^{19}$.

\section{Conclusiones}

Según la literatura revisada en los últimos 10 años respecto al trasplante facial en el periodo preoperatorio, aunque no hay un consenso respecto a los criterios de elección de los pacientes donantes y receptores, ya hay instituciones con protocolos establecidos. En cuanto a la población pediátrica, el procedimiento aún no seharealizado, dadolos efectos de la terapia inmunosupresora sobre el crecimiento y desarrollo en este grupo etáreo. El periodo intraoperatorio se encuentra descrito de manera clara en la literatura, y respecto al postoperatorio se conoce la terapia inmunosupresora, sin embargo, los actuales fármacos utilizados tienen importantes efectos secundarios y su costo es elevado, lo cual es una limitación, junto a la infraestructura y equipo humano necesario para llevar a cabo el procedimiento; se espera que a medida que se extienda la práctica, estos disminuyan y sea más accesible para los sistemas de salud. Finalmente, el TF es un procedimiento relativamente nuevo en el cual aún hay áreas por explorar y posibles desenlaces y complicaciones de las cuales no se tiene mucha certeza, sin embargo, se muestra como una alternativa prometedora a los métodos de reconstrucción utilizados para el manejo de lesiones faciales complejas.

\section{Agradecimientos}

Al grupo y semillero de investigación de Cirugía Plástica, Reconstructiva y Estética PLASTICUN, y a los integrantes del departamento de Cirugía Plástica de la Universidad Nacional de Colombia.

\section{Conflictos de interés}

Los autores declaran no tener conflictos de interés de ningún tipo.

\section{Referencias bibliográficas}

1. Sosin M, Rodriguez ED. The Face Transplantation Update: 2016. Plast Reconstr Surg. 2016;137(6):1841-50.

2. Cammarata MJ, Jethanamest D, Rodriguez ED. Otologic considerations in a full face transplant recipient. Laryngoscope. 2018;129(9): 2008-11.

3. Topçu Ç, Uysal H, Özkan Ö, Özkan Ö, Polat Ö, Bedeloğlu M, et al. Recovery of facial expressions using functional electrical stimulation after full-face transplantation. J Neuroeng Rehabil. 2018;15(15):1-7.

4. Sweeney N, Allen K, Miller B, Nolan T, Sheerin K. Perioperative Nursing Management of Donor and Recipient Patients Undergoing Face Transplantation. AORN J [Internet]. 2017;106(1):8-19. Disponible en: http://dx.doi.org/10.1016/j.aorn.2017.05.004

5. Bharadia D, Sinha I, Pomahac B. Role of Facial Vascularized Composite Allotransplantation in Burn Patients. Clin Plast Surg [Internet]. 2017;44(4):857-64. Disponible en: http://dx.doi. org/10.1016/j.cps.2017.05.017

6. Wallins JS, Chandawarkar AA, Dobry A, Diaz-Siso JR, Bueno EM, Caterson EJ, et al. Craniofacial measurements of donors and recipients correlate with aesthetic outcome in virtual face transplantation. Plast Reconstr Surg. 2015;3(5):1-7.

7. Keener AB. Saving face: The search for alternatives to life-long immunosuppression for face transplants. Nat Med [Internet]. 2016;22(5):448-9. Disponible en: http://dx.doi.org/10.1038/ nm0516-448

8. Aycart MA, Pomahac B. Discussion. Plast Reconstr Surg [Internet] 2016;138(5):1080-1. Disponible en: https://doi.org/10.1097/ PRS.0000000000002717

9. Lee J. Face transplantation for the blind: more than being blind in a sighted world. J Med Ethics 2018;44(6):361-5.

10. Hashem AM, Hoffman GS, Gastman B, Bernard S, Djohan $\mathrm{R}$, et al. Establishing the Feasibility of Face Transplantation in Granulomatosis With Polyangiitis. Am J Transplant. 2016;16(7):2213-23.

11. Oser ML, Nizzi MC, Zinser JL, Turk M, Epstein R, et al. Quality of Life and Psychosocial Functioning 2 Years Following Facial Transplantation. Psychosomatics [Internet]. 2018;59(6):591-600. Disponible en: https://doi.org/10.1016/j.psym.2018.04.005

12. Cogliandro A, Barone M, Salzillo R, Persichetti P. Quality of Life after Face Transplantation. Plast Reconstr Surg [Internet]. 2017;140(5):757e. Disponible en: https://doi.org/10.1097/ PRS.0000000000003818

13. Sosin M, Ceradini DJ, Levine JP, Hazen A, Staffenberg DA, Saadeh PB, et al. Total face, eyelids, ears, scalp, and skeletal subunit transplant: A reconstructive solution for the full face and total scalp burn. Plast Reconstr Surg [Internet]. 2016;138(1):205-19. Disponible en: https://doi.org/10.1097/PRS.0000000000002322

14. Caterson EJ, Diaz-Siso JR, Shetye P, Junker JPE, Bueno EM, et al. Craniofacial principles in face transplantation. J Craniofac Surg. 2012;23(5):1234-8.

15. Sedaghati-nia A, Gilton A, Liger C, Binhas M, Cook F, AitMammar B, et al. Anaesthesia and intensive care management of face transplantation. Br J Anaesth. 2013:111(4):600-6.

16. Diaz-Siso JR, Plana NM, Schleich B, Irving H, Gelb BE, et al. Novel Donor Transfer Algorithm for Multiorgan and Facial Allograft Procurement. Am J Transplant. 2017;17(9):2496-7.

17. Kiwanuka H, Aycart MA, Bueno EM, Alhefzi M, Krezdorn N, Pomahac B. Patient Recruitment and Referral Patterns in Face Transplantation. Plast Reconstr Surg. 2016;138(1):224-31.

18. Tasigiorgos S, Kollar B, Krezdorn N, Bueno EM, Tullius SG, Pomahac B. Face transplantation-current status and future developments. Transpl Int. 2018;31(7):677-88.

19. Nguyen LL, Naunheim MR, Hevelone ND, Diaz-Siso JR, Hogan JP, Bueno EM, et al. Cost analysis of conventional face reconstruction versus face transplantation for large tissue defects. Plast Reconstr Surg. 2015;135(1):260-7.

20. Shanmugarajah K, Hettiaratchy S, Butler PEM. Facial transplantation. Curr Opin Otolaryngol Head Neck Surg [Internet]. 2012;20(4):291-7. Disponible en: http://content. wkhealth.com/linkback/openurl?sid=WKPTLP:landingpage\& an $=00020840-201208000-00012$

21. Aycart MA, Alhefzi M, Kueckelhaus M, Krezdorn N, Bueno EM, Caterson EJ, et al. A Retrospective Analysis of Secondary Revisions after Face Transplantation: Assessment of Outcomes, Safety, and Feasibility. Plast Reconstr Surg. 2016;138(4):690e-701e.

22. Pomahac B, Bueno EM, Sisk GC, Pribaz JJ. Current principles of facial allotransplantation: The Brigham and Women's Hospital experience. Plast Reconstr Surg. 2013;131(5):1069-76.

23. Diaz-Siso JR, Rodriguez ED. Discussion. Plast Reconstr Surg. 2015;137(1):223e-224e.

24. Marchac A, Kuschner T, Paris J, Picard A, Vazquez MP, Lantieri L. Ethical issues in pediatric face transplantation: Should we perform face transplantation in children? Plast Reconstr Surg. 
Méndez-Cárdenas JG, Nieto-Fernández D, Erazo-Arteaga MC, Pineda-López DM,

Quintero-Cuervo DY, Santamaría-Losada PA, Ruiz-Moreno CE, Nieto-Bayona MA, Leuro-

2016;138(2):449-54.

25. Devauchelle BL, Testelin SR, Davrou J, Neiva C, Lengele BG, Dubernard JM, et al. Face graft? Extrapolation of facial allotransplantation to children. J Cranio-Maxillofacial Surg [Internet]. 2016;44(8):925-33. Disponible en: http://dx.doi. org/10.1016/j.jcms.2016.03.006

26. Dorante MI, Tasigiorgos S, Pomahac B. Pediatric face transplantation: a viable option?. Curr Opin Organ Transplant. 2018;23(5): 598-604.

27. Coffman KL. Psychological and Psychosocial Aspects of Face Transplantation. In: Sher Y, Maldonado JR, editors. Psychosocia Care of End-Stage Organ Disease and Transplant Patients. 19th ed. California: Springer; 2019. 353-363.

28. Park J, Yim S, Eun SC. Experimental design for composite face transplantation. J Craniofac Surg. 2016;27(4):843-5.

29. Duisit J, Debluts D, Behets C, Gerdom A, Vlassenbroek A, Coche E, et al. Porcine ear: A new model in large animals for the study of facial subunit allotransplantation. JPRAS Open. 2017;12:47-58.

30. Eun SC. Facial transplantation surgery. Arch Plast Surg 2014;41(2):174-80.

31. Shockcor N, Buckingham B, Hassanein W, Haririan A, Roberts SM, Nam AJ, et al. End Stage Renal Disease as a Complication of Face Transplant. Transplant Direct. 2018;102:S434.

32. Wall A, Bueno E, Pomahac B, Treister N. Intraoral features and considerations in face transplantation. Oral Dis. 2016;22(2):93103.

33. Lantieri L, Hivelin M, Audard V, Benjoar MD, Meningaud JP, Bellivier F, et al. Feasibility, reproducibility, risks and benefits of face transplantation: A prospective study of outcomes. Am J Transplant. 2011;11(2):367-78.

34. Siemionow M, Papay F, Alam D, Bernard S, Djohan R, Gordon C, et al. Near-total human face transplantation for a severely disfigured patient in the USA. Lancet. 2009;374(9685):203-9.
35. McKay TE, Balou M, Kao DJ, Ho DJ, Cohen J, Rodriguez ED. Poster 71 New Frontiers: Inpatient Comprehensive Rehabilitation After Full Face Transplantation: A Case Report. Pm\&R [Internet]. 2016;8(9):S184. Disponible en: http://dx.doi.org/10.1016/j. pmrj.2016.07.114

36. Perry BJ, Richburg BD, Pomahac B, Bueno EM, Green JR. The Effects of Lip-Closure Exercise on Lip Strength and Function Following Full Facial Transplantation: A Case Report. Am J Speech Lang Pathol [Internet]. 2017;26(2S):682-686. Disponible en: https://doi.org/10.1044/2017_AJSLP-16-0101

37. Rifkin WJ, Manjunath A, Kimberly LL, Plana NM, Kantar RS, Bernstein GL, et al. Long-distance care of face transplant recipients in the United States. J Plast Reconstr Aesthetic Surg [Internet]. 2018;71(10):1383-1391. Disponible en: https://doi. org/10.1016/j.bjps.2018.05.019

38. "Social anonymity": The ethics of facial transplantation. Br Dent J. 2016;221(3):126-126.

39. Chang G, Pomahac B. Psychosocial Changes Six Months after Face Transplantation. Psychosomatics [Internet]. 2013;54(4):367371. Disponible en: http://dx.doi.org/10.1016/j.psym.2012.07.012

40. Giatsidis G, Sinha I, Pomahac B. Reflections on a decade of face transplantation. Ann Surg. 2017;265(4):841-846.

41. Lantieri L, Grimbert P, Ortonne N, Suberbielle C, Bories D, GilVernet S, et al. Face transplant: long-term follow-up and results of a prospective open study. Lancet. 2016;388(10052):1398-1407.

42. Siemionow M, Gatherwright J, Djohan R, Papay F. Cost analysis of conventional facial reconstruction procedures followed by face transplantation. Am J Transplant. 2011;11(2):379-385.

43. Fischer S, Diehm Y, Dorante MI, Kotsougiani D, Kueckelhaus M, Alhefzi M, et al. Software-based video analysis of functional outcomes of face transplantation. Microsurgery [Internet]. 2018;39(1):53-61. Disponible en: http://doi.wiley.com/10.1002/ micr.30360 